\title{
Factors Impacting Mortality in Geriatric Patients with Acute Spine Fractures: A 12-Year Study of 613 Patients in Singapore
}

\author{
En Loong Soon ${ }^{1,2}$, Adriel Zhijie Leong ${ }^{1,2}$, Jean Chiew ${ }^{1,2}$, \\ Arun-Kumar Kaliya-Perumal ${ }^{1}$, Chun Sing $\mathrm{Yu}^{1,2}$, Jacob Yoong-Leong $\mathrm{Oh}^{1,2}$ \\ ${ }^{I}$ Division of Spine, Department of Orthopaedic Surgery, Tan Tock Seng Hospital, Singapore \\ ${ }^{2}$ Lee Kong Chian School of Medicine, Singapore
}

Study Design: Retrospective database analysis.

Purpose: To identify risk factors that predict mortality following acute spine fractures in geriatric patients of Singapore.

Overview of Literature: Acute geriatric spinal fractures contribute significantly to local healthcare costs and hospital admissions. However, geriatric mortality following acute spine fractures is scarcely assessed in the Asian population.

Methods: Electronic records of 3,010 patients who presented to our hospital's emergency department and who were subsequently admitted during 2004-2015 with alleged history of traumatic spine fractures were retrospectively reviewed, and 613 patients (mean

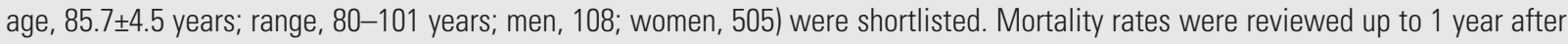
admission and multivariate analyses were performed to identify independent risk factors correlating with mortality.

Results: Women were more susceptible to spine fractures $(82.4 \%)$, with falls $(77.8 \%)$ being the most common mechanism of injury. Mortality rates were $6.0 \%, 8.2 \%$, and $10.4 \%$ at 3,6 , and 12 months, respectively. The most common causes of death at all 3 time points were pneumonia and ischemic heart disease. Based on the multivariate analysis at 1-year follow-up, elderly women had a lower mortality rate compared to men $(p<0.001)$; mortality rates increased by $6.3 \%(p=0.024)$ for every 1 -year increase in the patient's age; and patients with an American Spinal Injury Association (ASIA) score of A-C had a much higher mortality rate compared to those with an ASIA score of $\mathrm{D}-\mathrm{E}(p<0.001)$.

Conclusions: An older age at presentation, male sex, and an ASIA score of A-C were identified as independent factors predicting increased mortality among geriatric patients who sustained acute spine fractures. The study findings highlight at-risk groups for acute spine fractures, thereby providing an opportunity to develop strategies to increase the life expectancy of these patients.

Keywords: Accidental falls; Mortality; Spinal cord injuries; Spinal fractures; Spinal injuries

\section{Introduction}

In Asian countries, the proportion of people older than
65 years has been increasing rapidly. According to official statistics regarding population trends, the percentage of elderly people in Singapore has grown from $6 \%$ to $12.4 \%$

Received Sep 10, 2018; Revised Nov 5, 2018; Accepted Nov 20, 2018

Corresponding author: Jacob Yoong-Leong Oh

Division of Spine, Department of Orthopaedic Surgery, Tan Tock Seng Hospital, 11 Jalan Tan Tock Seng, Singapore 308433

Tel: +65-6257-7000, Fax: +65-6357-7715, E-mail: Jacob_oh@yahoo.com 
in the past 15 years, and this percentage is only expected to increase in the years ahead. As the population ages, the frequency of acute geriatric spinal trauma increases exponentially [1-4]; occurs despite low energy mechanism, such as minor falls [5-7], which increase proportional to the individual's age $[8,9]$.

As individuals age, their posture, protective reflexes, lower extremity proprioception, vision, and vestibular system functions deteriorate. This deterioration coupled with other age-related comorbidities, such as osteoarthritis of the joints and neuromuscular or neurological disorders, has the potential to reduce mobility and postural stability, thereby predisposing them to falls [9]. In addition to having an increased risk of falls, the frequency of osteoporosis in individuals of this age group is high, predisposing them to hip, spine, or distal radius fractures [10].

Vertebral fractures cause significant complications, including chronic back pain, height loss, kyphosis, and activity limitations [11-13]. These complications might be associated with increased mortality [14]. As such, the significance of the association between spinal trauma and mortality cannot be underestimated [15]. Given the significant costs of healthcare and rehabilitation [4], this study aimed to delineate trends among geriatric patients in Singapore who have sustained acute spinal trauma and to provide mortality data for an important demographic group that has scarcely been assessed locally.

\section{Materials and Methods}

After obtaining approval (IRB approva no., 2017/00419) from the Domain Specific Review Board (DSRB), National Healthcare Group (NHG), Singapore, the electronic records of patients who presented to our hospital's emergency department and who were subsequently admitted between 2004 and 2015 with a history of traumatic spine fractures were retrospectively reviewed. We shortlisted patients older than 80 years of age who had sustained an acute single or multilevel spinal compression fracture (with or without spinal cord injury), confirmed by radiology. We excluded those patients with fractures secondary to infections or neoplasms.

Included patients were generally received in the emergency department and evaluated. Their history regarding the exact mechanism of injury was recorded. A complete neurological examination was performed to categorize patients according to their American Spinal Injury Asso- ciation (ASIA) score. Patients were sent for initial X-rays, computed tomography (CT), and magnetic resonance imaging (MRI) scans to find evidence of acute spinal compression fractures. The presence of an acute fracture was confirmed by a senior spine surgeon and the radiologist. The presence of concomitant chronic osteoporotic fractures in the rest of the spine or other bones was also noted. Once a diagnosis was achieved, emergency surgery, elective surgery, or conservative management was planned depending on the nature of the injury, its severity, and the presence of comorbidities.

Demographic data including age, sex, ethnicity, date of admission, mechanism of injury, comorbidities at admission (diabetes mellitus, hypertension, hyperlipidemia, ischemic heart disease, or previous stroke), activities of daily living (ADL) status, ambulation status, mode of treatment, and length of hospital stay were collected from our computerized patient support system. Radiological data, including X-ray, CT, and MRI images along with their reports were reviewed from our picture archive and communication system to note the level of the fractures. The date of death and the exact cause of death were obtained with approval from the National Registry of Disease Office.

All the statistical analyses were performed using IBM SPSS Statistics for Windows ver. 24.0 (IBM Corp., Armonk, NY, USA). A $p$-value of $<0.05$ was considered statistically significant. Linear relationships between age and length of hospital stay were analyzed by calculating Pearson's correlation coefficient $(r)$. The strength of the correlation was considered stronger when the ' $r$ ' value was close to 1 . Mortality rates at 3,6, and 12 months were calculated, and the cause of death at each time point was analyzed. Multivariate Cox regression for 1-year overall survival was performed. Hazard ratios were calculated for variables significantly correlating with mortality. This study was performed in accordance with the ethical standards stated in the most recent version of the 1964 Declaration of Helsinki or comparable ethical standards. The requirement of informed consent for patient inclusion was waived by the NHG DSRB.

\section{Results}

Electronic records of 3,010 patients who presented to our emergency department with acute spine fracture and who were subsequently admitted were reviewed; of which, 613 
Table 1. Demographics and general characteristics of study population ( $n=613$ )

\begin{tabular}{|c|c|}
\hline Variable & No. of patients (\%) \\
\hline \multicolumn{2}{|l|}{ Age (yr) } \\
\hline $80-85$ & $337(54.98)$ \\
\hline $86-90$ & $180(29.36)$ \\
\hline $91-95$ & $76(12.40)$ \\
\hline $96-100$ & $18(2.94)$ \\
\hline$>100$ & $2(0.33)$ \\
\hline \multicolumn{2}{|l|}{ Sex } \\
\hline Male & $108(17.62)$ \\
\hline Female & 505 (82.38) \\
\hline \multicolumn{2}{|l|}{ Ethnicity } \\
\hline Chinese & $563(91.84)$ \\
\hline Malay & $26(4.24)$ \\
\hline Indian & $18(2.94)$ \\
\hline Others & $6(0.98)$ \\
\hline \multicolumn{2}{|l|}{ Presence of comorbidities } \\
\hline Hypertension & $435(71.00)$ \\
\hline Diabetes mellitus & $168(27.40)$ \\
\hline Hyperlipidaemia & $339(55.30)$ \\
\hline Ischemic heart disease & $150(24.50)$ \\
\hline Cerebrovascular disease & $98(16.00)$ \\
\hline Presence of prior osteoporotic fracture & $258(42.10)$ \\
\hline \multicolumn{2}{|l|}{ Mechanism of injury } \\
\hline Falls & $477(77.81)$ \\
\hline Sudden back pain & $112(18.27)$ \\
\hline Others & $24(3.92)$ \\
\hline \multicolumn{2}{|l|}{ Type of injury } \\
\hline Spinal cord involvement & $9(1.47)$ \\
\hline Isolated cervical compression fractures & $15(2.45)$ \\
\hline Isolated thoracic compression fractures & $149(24.30)$ \\
\hline $\begin{array}{l}\text { Multilevel compression fractures involving } \\
\text { the thoracic and lumbar levels }\end{array}$ & $91(14.80)$ \\
\hline Isolated lumbar compression fractures & $272(44.37)$ \\
\hline Isolated sacral fractures & $60(9.79)$ \\
\hline Others & $17(2.77)$ \\
\hline \multicolumn{2}{|l|}{ American Spinal Injury Association score } \\
\hline A & $4(0.65)$ \\
\hline B & 0 \\
\hline C & $3(0.49)$ \\
\hline $\mathrm{D}$ & $171(27.90)$ \\
\hline E & $435(71.00)$ \\
\hline \multicolumn{2}{|l|}{ Treatment } \\
\hline Surgery & 28 (4.57) \\
\hline Conservative & $585(95.43)$ \\
\hline
\end{tabular}

patients (mean age, $85.7 \pm 4.5$ years; range, $80-101$ years; men, 108; women, 505) met our selection criteria. Their demographic characteristics, comorbidities, mechanism of injury, level of fracture, and mode of treatment were tabulated (Table 1). This sample revealed a preponderance of women $(82.38 \%)$ among older people who sustain acute spine fractures compared with men (17.62\%). Falls in the community accounted for the most common mechanism of injury (77.81\%), with the remaining $18.27 \%$ experiencing a traumatic sudden back pain that prompted their presentation.

The prefracture ADL status was categorized into independent (464 patients, 75.7\%) and assisted (149 patients, $24.3 \%)$. Ambulation status was categorized into nonambulant/needing assistance (42 patients, 6.8\%), home ambulant (116 patients, $18.9 \%$ ), and community ambulant (455 patients, $74.2 \%$ ). The sample predominantly included patients with osteoporotic compression fractures of the spine $(85.92 \%)$ without spinal cord injury. Only a small percentage $(1.47 \%)$ of patients had spinal cord signal changes on MRI. More than four in five fractures $(83.47 \%)$ involved the thoracic and lumbar levels, with $47.4 \%$ involving the thoracolumbar junction (T12-L1).

Table 2. Mortality rates and top 3 causes of death $(n=613)$

Variable No. of patients (\%)

Mortality rates

$\begin{array}{ll}\text { At } 3 \text { mo } & 36(6.0) \\ \text { At } 6 \text { mo } & 50(8.2) \\ \text { At } 1 \text { yr } & 63(10.4)\end{array}$

Causes of death at 3 mo

\begin{tabular}{lr} 
Pneumonia & $9(25.0)$ \\
\hline Ischemic heart disease & $7(19.4)$ \\
\hline Urinary tract infection (urosepsis) & $5(13.9)$ \\
\hline Others & $15(41.7)$ \\
Causes of death at 6 mo & $14(28.0)$ \\
\hline Pneumonia & $11(22.0)$ \\
Ischemic heart disease & $6(12.0)$ \\
\hline Metastatic cancer & $19(38.0)$ \\
\hline Others &
\end{tabular}

Causes of death at $1 \mathrm{yr}$

\begin{tabular}{ll} 
Pneumonia & $18(28.6)$ \\
\hline Ischemic heart disease & $14(22.2)$ \\
Metastatic cancer & $10(15.9)$ \\
\hline Others & $21(33.3)$ \\
\hline
\end{tabular}


Only $4.57 \%$ of patients ultimately underwent surgery, which was indicated mostly for pain due to compression fractures at the thoracolumbar junction. All the remaining patients $(95.43 \%)$ were conservatively managed. In more than $50 \%$ of those who underwent surgery, a single-level percutaneous vertebroplasty was performed after a failed conservative approach for a thoracolumbar compression fracture. We also noticed that $42.1 \%$ of the patients had sustained prior osteoporotic fractures involving the spine, hip, and/or forearm. The average length of hospital stay was $12.2 \pm 32$ days and was not influenced by the age of the patients $(r=-0.034)$.

Stratification of the data at 3 months, 6 months, and 1 year revealed the mortality rates and top three causes of death at each time point (Table 2). The mortality rates at 3 , 6 , and 12 months were $6 \%, 8.2 \%$, and $10.4 \%$, respectively. At 3 months, the top three ranked causes of death were pneumonia, ischemic heart disease, and urinary tract infection. Pneumonia, ischemic heart disease, and metastatic cancer featured as the top three ranked causes of death at each period at 6 months and 1 year.

Multivariate Cox regression for 1-year overall survival was performed. Variables including age, sex, mobility status, medical comorbidities, presence of old compression fractures, level of the spinal injury, ASIA score, and surgery performed were all found to influence mortality. Next, forward stepwise (likelihood ratio) selection was performed, which identified sex, age, and ASIA score, which remained in the final model and were independently correlated with mortality with statistical significance, thus predicting overall survival at 1 year (Table 3 ).

We found that elderly women had a lower mortality rate compared with men $(p<0.001)$ within the 1 -year followup period. Considering age as a continuous variable with a hazard ratio of 1.063 , we found that mortality rates increased by $6.3 \%(p=0.024)$ for every 1 -year increase in age. In addition, the ASIA score $(\mathrm{A}-\mathrm{C})$ had a hazard ratio of 12.36, indicating that patients with an ASIA score of A$C$ had a much higher mortality rate $(p<0.001)$ compared with ASIA D-E patients.

\section{Discussion}

This study describes, over the course of 12 years, the epidemiology of elderly patients (age $>80$ years) who had sustained acute spine fractures and presented to a Singapore tertiary hospital. The results provide practicing clinicians with valuable information about the mechanism of injury, the level of spine fracture, and the patient demographics. The majority were Chinese $(91.84 \%)$, followed by Malay (4.24\%) and Indian (2.94\%) patients, mirroring our overall national demographic of $74.3 \%, 13.4 \%$, and $9 \%$, respectively, across all age groups [16]; signifying that acute spinal trauma relatively equally affects the three main ethnicities of our country's geriatric population.

We noticed that women were predisposed to acute spine fractures, which were predominantly due to minor falls [17]. This result is unsurprising, given a larger proportion of our national population older than 65 years are women [18]. Women also have a higher prevalence of osteoporosis after menopause [19], and older patients have an increased frequency of falls [20]. Interventions to reduce acute geriatric spinal trauma can therefore be better channeled toward preventing falls, reducing osteoporosis rates, and targeting women [21].

Thoracolumbar junction (T12-L1) fractures comprised the bulk of spinal injuries in our sample. The transition from the less mobile thoracic spine, with its adjoining sternum and ribs, to the more dynamic lumbar spine is known to subject the thoracolumbar region to greater biomechanical stress, accounting for the location of the injuries sustained [22,23]. Interestingly, those with a prior osteoporotic fracture had a greater chance of sustaining a subsequent spine fracture, with $42.1 \%$ of the patients within our cohort having sustained a prior osteoporotic fracture [24,25].

The most common cause of death after acute spine fractures was pneumonia, followed by ischemic heart disease. Patients with acute spine fractures are likely to have greater pain, leading to immobility and thereby predisposing them to pneumonia $[26,27]$. They are also susceptible to

Table 3. Multivariate Cox regression for 1-year overall survival

\begin{tabular}{lcc} 
Variable & $p$-value & Hazard ratio (95\% confidence interval) \\
Female sex & $<0.001$ & $0.340(0.200-0.579)$ \\
Age at admission & 0.024 & $1.063(1.008-1.120)$ \\
American Spinal Injury Association score (A-C) & $<0.001$ & $12.363(4.727-32.340)$ \\
\hline
\end{tabular}


other pulmonary problems and are at an increased risk of subsequent nonvertebral problems [28]. Our results showed that approximately one in 10 patients died at 6 months and at 1 year following an acute spine fracture. Therefore, it is important to prevent falls leading to acute spine fractures and subsequent morbidity.

Based on our analysis, the risk factors associated with increased mortality at 1 year include the male sex, an older age, and an ASIA score of A-C. Although older age at presentation meant that patients were closer to their natural life expectancy, the presence of acute spine fractures was associated with a higher than expected mortality rate. An ASIA score of A-C indicates severe neurological compromise due to spinal cord injury. With its expected resultant morbidity, there is a greater likelihood of mortality.

Of the patients who underwent surgery, $80 \%$ were patients with an ASIA score of D-E who underwent percutaneous vertebroplasty. We postulate that these patients performed better after surgery, given the procedure led to a faster recovery, increased stability, pain relief, and greater mobility compared with patients who were conservatively managed $[29,30]$. It was also likely that fitter patients with a lower perioperative risk were selected for surgery. These individuals likely had lesser comorbidities, better pre-existing function, and a resultant decrease in mortality rates compared with patients who did not have these advantages.

The main limitation of our study is that our sample population might not have been fully representative of the geriatric population with acute spine fractures. A significant number of patients with acute compression fractures of the spine are treated in an outpatient setting; thus, they were not included in this study. In addition, we did not have data on the functional status of patients throughout the follow-up. This information is important, given immobility is directly linked with the onset of pneumonia in functionally compromised patients. Despite these limitations, we believe our study to be of clinical importance and useful for predicting prognoses.

\section{Conclusions}

We found that approximately one in 10 patients older than 80 years of age died at 6 months and 1 year after sustaining an acute spine fracture. We also found that (1) elderly women had a lower mortality rate compared with men; (2) the mortality rate increased by $6.3 \%$ for every 1 -year increase in age; and (3) an ASIA score of A-C had a much higher mortality rate compared with ASIA D-E patients. An older age at presentation, the male sex, and an ASIA score of $\mathrm{A}-\mathrm{C}$ were independent factors predictive of increased mortality among geriatric patients who sustained acute spine fractures. Considering the associated morbidity and mortality, preventing spine fractures in the elderly is a cornerstone in maintaining their quality of life.

\section{Conflict of Interest}

No potential conflict of interest relevant to this article was reported.

\section{References}

1. Daneshvar P, Roffey DM, Brikeet YA, Tsai EC, Bailey CS, Wai EK. Spinal cord injuries related to cervical spine fractures in elderly patients: factors affecting mortality. Spine J 2013;13:862-6.

2. Jabbour P, Fehlings M, Vaccaro AR, Harrop JS. Traumatic spine injuries in the geriatric population. Neurosurg Focus 2008;25:E16.

3. Jawa RS, Singer AJ, Rutigliano DN, et al. Spinal fractures in older adult patients admitted after low-level falls: 10-year incidence and outcomes. J Am Geriatr Soc 2017;65:909-15.

4. Tian Y, Zhu Y, Yin B, et al. Age- and gender-specific clinical characteristics of acute adult spine fractures in China. Int Orthop 2016;40:347-53.

5. Wang H, Coppola M, Robinson RD, et al. Geriatric trauma patients with cervical spine fractures due to ground level fall: five years experience in a level one trauma center. J Clin Med Res 2013;5:75-83.

6. Golob JF Jr, Claridge JA, Yowler CJ, Como JJ, Peerless JR. Isolated cervical spine fractures in the elderly: a deadly injury. J Trauma 2008;64:311-5.

7. Bhattacharya B, Maung A, Schuster K, Davis KA. The older they are the harder they fall: injury patterns and outcomes by age after ground level falls. Injury 2016;47:1955-9.

8. Centers for Disease Control and Prevention (CDC). Fatalities and injuries from falls among older adults: United States, 1993-2003 and 2001-2005. MMWR Morb Mortal Wkly Rep 2006;55:1221-4.

9. Fuller GF. Falls in the elderly. Am Fam Physician 2000;61:2159-68,2173-4. 
10. Tian L, Yang R, Wei L, et al. Prevalence of osteoporosis and related lifestyle and metabolic factors of postmenopausal women and elderly men: a crosssectional study in Gansu province, Northwestern of China. Medicine 2017;96:e8294.

11. Yeung YK, Ho ST. Delayed neurological deficits after osteoporotic vertebral fractures: clinical outcomes after surgery. Asian Spine J 2017;11:981-8.

12. Ross PD. Clinical consequences of vertebral fractures. Am J Med 1997;103:30S-42S.

13. Nevitt MC, Ross PD, Palermo L, Musliner T, Genant $\mathrm{HK}$, Thompson DE. Association of prevalent vertebral fractures, bone density, and alendronate treatment with incident vertebral fractures: effect of number and spinal location of fractures: the Fracture Intervention Trial Research Group. Bone 1999;25:613-9.

14. Kado DM, Browner WS, Palermo L, Nevitt MC, Genant HK, Cummings SR. Vertebral fractures and mortality in older women: a prospective study: study of Osteoporotic Fractures Research Group. Arch Intern Med 1999;159:1215-20.

15. Center JR, Nguyen TV, Schneider D, Sambrook PN, Eisman JA. Mortality after all major types of osteoporotic fracture in men and women: an observational study. Lancet 1999;353:878-82.

16. Lim RB, Zheng H, Yang Q, Cook AR, Chia KS, Lim WY. Ethnic and gender specific life expectancies of the Singapore population, 1965 to 2009: converging, or diverging? BMC Public Health 2013;13:1012.

17. Yeo YY, Lee SK, Lim CY, Quek LS, Ooi SB. A review of elderly injuries seen in a Singapore emergency department. Singapore Med J 2009;50:278-83.

18. Yong V, Saito Y, Chan A. Gender differences in health and health expectancies of older adults in Singapore: an examination of diseases, impairments, and functional disabilities. J Cross Cult Gerontol 2011;26:189203.
19. Lau EM. Epidemiology of osteoporosis. Best Pract Res Clin Rheumatol 2001;15:335-44.

20. Dionyssiotis Y. Analyzing the problem of falls among older people. Int J Gen Med 2012;5:805-13.

21. Lee A, Lee KW, Khang P. Preventing falls in the geriatric population. Perm J 2013;17:37-9.

22. Kim BG, Dan JM, Shin DE. Treatment of thoracolumbar fracture. Asian Spine J 2015;9:133-46.

23. Rajasekaran S, Kanna RM, Shetty AP. Management of thoracolumbar spine trauma: an overview. Indian J Orthop 2015;49:72-82.

24. Gehlbach S, Saag KG, Adachi JD, et al. Previous fractures at multiple sites increase the risk for subsequent fractures: the Global Longitudinal Study of Osteoporosis in Women. J Bone Miner Res 2012;27:645-53.

25. Huntjens KM, Kosar S, van Geel TA, et al. Risk of subsequent fracture and mortality within 5 years after a non-vertebral fracture. Osteoporos Int 2010;21:2075-82.

26. Kim B, Kim J, Jo YH, et al. Risk of pneumonia after vertebral compression fracture in women with low bone density: a population-based study. Spine 2018;43:E830-5.

27. Wawruch M, Krcmery S, Bozekova L, et al. Factors influencing prognosis of pneumonia in elderly patients. Aging Clin Exp Res 2004;16:467-71.

28. van der Jagt-Willems HC, Vis M, Tulner CR, et al. Mortality and incident vertebral fractures after 3 years of follow-up among geriatric patients. Osteoporos Int 2013;24:1713-9.

29. Kaliya-Perumal AK, Lin TY. Clinical outcomes of percutaneous vertebroplasty for selective single segment dorsolumbar vertebral compression fractures. J Clin Orthop Trauma 2018;9:S140-4.

30. Chen LH, Lai PL, Chen WJ. Current status of vertebroplasty for osteoporotic compression fracture. Chang Gung Med J 2011;34:352-9. 